\title{
LA INCORPORACIÓN DE LOS FONDOS DE CONOCIMIENTO E IDENTIDAD EN LA ESCUELA NORMAL DE TEXCOCO, MÉXICO: LAS CREENCIAS DE LOS DOCENTES EN FORMACIÓN' ${ }^{1}$
}

\author{
David Subero Tomás \\ Universidad de Girona \\ Luis Fernando Brito Rivera \\ Profesor de la Escuela Normal de Texcoco (México) \\ Moisés Esteban-Guitart \\ Universidad de Girona
}

\begin{abstract}
RESUMEN: Existe un consenso acerca del carácter situado y distribuido que tienen hoy los aprendizajes, y con ello la necesidad de establecer continuidades educativas entre el contexto social de los estudiantes de origen culturalmente diverso y la propia escuela. En este sentido, el objetivo de este artículo es hacer emerger las creencias iniciales de los docentes en formación sobre la familia y la escuela a través de tres sesiones de grupos focales. Participan 16 normalistas, hombres y mujeres. Los docentes expresan una falta de relación entre los contextos educativos formales e informales y los contextos socioculturales del alumno, hecho que justifica la participación en el programa educativo "fondos de conocimiento e identidad" para modificar estas creencias. Las conclusiones presentadas forman parte del primer ciclo de una investigación basada en el diseño desarrollado en la formación inicial del profesorado de Escuelas Normales, México.
\end{abstract}

PALABRAS CLAVE: Fondos de conocimiento e identidad, creencias de los docentes, relación familia-escuela, formación inicial profesorado.

1. Los autores dedican este trabajo a todos los docentes en formación del segundo año de la licenciatura en educación secundaria con especialidad en historia (LES-Historia) de la Escuela Normal de Texcoco: Martha, Blanca, Susana, Hycel, Madeleim, Denisse, Nimbe, Paola, Mayra, Norma, Daniela, Iván, Giovanni, David, Aldahir, Jordan. Ustedes son parte esencial del futuro de la educación. Gracias por su apoyo y confianza. 


\title{
THE INCORPORATION OF THE FUNDS OF KNOWLEDGE AND IDENTITY IN A NORMAL SCHOOL OF TEXCOCO, MEXICO: THE BELIEFS OF THE TEACHERS-IN-TRAINING
}

\begin{abstract}
General consensus has been reached about the localized and distributed nature of nowadays' education, together with the need to establish educational links between the social and cultural context of the students, and the school. Following these premises, the aim of the present paper is to promote the initial beliefs of teachers in training about the family and the schools throughout three focus group sessions. The participants were 16 teachers-in-training, both men and women. The teachers expressed there is a lack of congruency between formal and informal educational contexts, and the students' sociocultural background, which justifies the engagement in the "funds of knowledge and identity", in order to modify the teachers' beliefs. The conclusions presented in this study are part of the first cycle of a research based on the design developed for the initial training of teachers in "Escuelas Normales", Mexico.
\end{abstract}

KEYWORDS: Funds of knowledge and identity, teachers' beliefs, homeschool relationships, initial teacher training.

Recibido: 20/08/2015

Aceptado: 17/06/2016

Correspondencia: David Subero Tomás, Universidad de Girona, Departamento de Psicología, Institut de Recerca Educativa, Facultad de Educación y Psicología, Plaça Sant Domènec, 9, 17071 Girona. Email: davidsubero@gmail.com.

\section{INTRODUCCIÓN}

Durante el periodo de diciembre de 2014 a mayo de 2015, y en el marco de una estancia de intercambio de experiencias académicas y de investigación educativa entre la Escuela Normal de Texcoco, Estado de México y la Universidad de Girona, España, se desarrolló un proyecto de investigación e innovación educativa con el objetivo de fortalecer el proceso de formación inicial del profesorado. El propósito más importante fue consolidar una secuencia didáctica ${ }^{2}$ (SD) que, fundamentada en los fondos de conocimiento e identidad de los alumnos de secundaria, pudiera generar aprendizajes significativos tanto en alumnos como en docentes. Es decir, generar continuidades tanto en la interacción entre los actores educativos como entre sus contextos de referencia.

2. En la investigación se denomina secuencia didáctica a la unidad mínima del proceso de enseñanza y aprendizaje al reunir: a) unas intenciones educativas; b) finalidades u objetivos delimitados; c) contenidos específicos que son objeto de enseñanza y aprendizaje; d) un conjunto de actividades de enseñanza y aprendizaje que se ponen en marcha en un determinado orden con el propósito de asegurar el aprendizaje de esos contenidos; e) criterios, procedimientos e instrumentos de evaluación que permiten valorar el grado en que los alumnos y alumnas han aprendido los contenidos en cuestión y han alcanzado las finalidades pretendidas (Coll, Onrubia y Mauri, 2008). 
En este artículo 3 se presentan los resultados de la primera de las tres etapas de investigación cuyo interés ha sido explorar las creencias que los docentes en formación tienen sobre sus alumnos, familias y comunidades en relación a la escuela y a la educación. En virtud de estos propósitos, los datos presentados han sido generados durante el primer ciclo de una investigación basada en el diseño con la intención de confirmar, refinar y conjuntar datos que justifiquen la implementación del programa "fondos de conocimiento e identidad" a través de la historia oral para planear un segundo y tercer ciclo de investigación. La segunda etapa de la investigación se enmarca en la capacitación y desarrollo de los "fondos de conocimiento e identidad" por parte de los docentes en formación en sus centros de prácticas de secundaria y, finalmente, la tercera fase se enmarca en la elaboración de las secuencias didácticas culturalmente inclusivas y la implementación de la unidad didáctica por parte de los docentes en formación en el salón de clases de secundaria con el objetivo de generar continuidades entre los procesos de enseñanza y aprendizaje.

La Escuela Normal de Texcoco es la institución educativa elegida para llevar a cabo el proyecto de investigación. Las Escuelas Normales mexicanas tienen la responsabilidad de formar de manera exclusiva a los profesores de nivel básico (Diario Oficial de la Federación, 2013). El estado Mexicano es el encargado en exclusividad de prestar los servicios para la formación de docentes a través de las escuelas normales, así como de los respectivos planes y programas de estudio que prescriben el perfil del docente al graduarse (Dirección General de Compilación y Consulta del Orden Jurídico Nacional, 2011). Cuantitativamente se tiene un total de 454 escuelas normales en todo el país, de estas 264 son públicas y 190 de carácter privado, la matrícula contabilizada a la fecha es de 127.240 alumnos y 18 . 253 profesores (Dirección General de Educación Superior para Profesionales de la Educación, 2014). Vista la gran responsabilidad que comporta la educación normalista en México, el gobierno mexicano a través de la SEP (Secretaría de Educación Pública) ha llevado a cabo la reforma 2012 con el objetivo de renovar la formación docente. Actualmente, son las especialidades en Licenciatura de Educación Secundaria aquellas que están a punto de implementar el nuevo plan educativo basado en el marco socioconstructivista, en contraposición a las tensiones y resistencias que reflejaba hasta ahora un modelo educativo calificado de tradicionalista. Iniciativas educativas como las que se plantean en dicho proyecto van en consonancia con los objetivos y principios de la reforma 2012 y se fundamentan como una oportunidad para afianzar nuevas actitudes y prácticas educativas en la formación del profesorado.

3. Este trabajo es producto de la primera parte de una investigación que se desarrolla entre la Escuela Normal de Texcoco, Estado de México y el Departamento de Psicología Educativa de la Universidad de Girona, España. Dichas actividades se basan en una estancia de colaboración e intercambio de experiencias académicas y profesionales entre el Prof. David Subero Tomás (alumno del programa de doctorado interinstitucional en psicología de la educación -DIPE- de la Universidad de Girona) y el Doc. Luis Fernando Brito Rivera (catedrático de la Escuela Normal de Texcoco; Doctor por el programa de Doctorado en Ciencias en Educación Agrícola Superior -DCEAS- de la Universidad Autónoma Chapingo y alumno del Doctorado Interinstitucional en Psicología de la Educación -DIPE- de la Universidad de Girona). 
Actualmente, nos encontramos en un momento donde las nuevas tecnologías y la presencia de internet amplían las perspectivas del espacio público del conocimiento (Castells, 1994; UNESCO, 2005). Siendo así, tanto los flujos de información como las posibilidades de conectividad, entre los distintos propósitos de orden personal, social, económico y de trabajo, son característicos del cambio experimentando en las esferas de la actividad humana (OECD, 2012). Dichas circunstancias se plantean así ya que el acceso a la información es más expedito que en otras épocas, por lo que es indispensable adquirir habilidades y competencias del siglo XXI para el manejo y gestión de la información (Tedesco, 2011; OCDE, 2010). Esto implica reconocer que los conocimientos y saberes son cambiantes y dinámicos ya que, en las condiciones de modernidad actuales, ningún conocimiento es definitivo en el sentido tradicional puesto que éste se encuentra distribuido (Cole y Engeström, 2001; Salomon, 2001) y situado (Lave, 2001; Lave y Wenger, 2003) en el sistema de actividad humana (Leontiev, 1984; Engeström, 2014).

La propuesta de investigación, que aquí se presenta en su primera etapa, plantea la necesidad de entender los procesos de aprendizaje desde la perspectiva de la llamada "nueva ecología del aprendizaje" (Coll, 2013; Barron, 2010), en donde los contextos de educación no formal cobran mayor relevancia para el desarrollo de los aprendizajes en la escuela. Esta situación conlleva producir nuevas formas de conectar las actividades educativas formales con los contextos de aprendizaje por los que transita el alumno. Lo que supone la recuperación del concepto de mesosistema (Bronfenbrenner, 1987) según el cual el potencial evolutivo de un determinado contexto o escenario de aprendizaje aumenta en la medida que sea capaz de generar continuidades educativas (lazos de confianza mutua y colaboración alrededor de objetivos comunes y compartidos) con otros escenarios educativos en los que la persona en desarrollo participa activamente. Este fenómeno modifica, de manera sustancial, la identidad de profesores y alumnos así como las funciones, roles, significados y sentidos de la actividad de enseñar y aprender.

Desde esta perspectiva, la escuela ha de crear nuevas posibilidades de acceso a las ideas, conversaciones, herramientas y redes sociales de los contextos informales de aprendizaje con el objetivo de enriquecer el desarrollo de las personas. Esto implica un replanteamiento importante sobre las funciones de la institución escolar, ya que el reto es la búsqueda de oportunidades de aprendizaje para que los alumnos fortalezcan sus intereses y satisfagan sus necesidades individuales contando con la responsabilidad compartida del centro escolar y del resto de los agentes sociales (Coll, 2010). Por ello, el interés fundamental del proyecto consiste en la reubicación y redefinición de la escuela dentro de la red de contextos de aprendizaje por la que transitan los alumnos. Específicamente sobre cómo generar un proceso educativo mediado por elementos socioculturales de alto significado y que, bajo el replanteamiento de la enseñanza desde la misma formación inicial del profesorado, pueda producir continuidades entre las prácticas y recursos culturales de los diferentes contextos de vida del alumnado.

Por lo anterior, lo que se plantea aquí es una propuesta educativa innovadora encaminada a generar mejores vías de inclusión educativa entre los contextos de vida de los alumnos y la escuela aproximándonos al marco teórico-metodológico de los "fondos de conocimiento e identidad" (Esteban-Guitart, 2014). Este marco 
busca superar la existencia de discontinuidades en la relación profesor-alumno, especialmente de aquellos estudiantes sub-representados por diversas razones (escasos ingresos, minoría étnica, clase social baja, etc.). Para solventar esta problemática, se propone poner en marcha un proceso educativo congruente con las necesidades de aprendizaje del alumnado así como de sus conocimientos, habilidades y destrezas emanadas de sus recursos y prácticas culturales. Esto nos permite concebir la educación como una actividad de inclusión e interactividad desde la base misma de la cultura e historia de vida del alumnado.

Los fondos de identidad son "aquellos artefactos, tecnologías o recursos, históricamente acumulados, culturalmente desarrollados y socialmente distribuidos y transmitidos, esenciales para la autodefinición, autoexpresión y autocomprensión de las personas" (Esteban-Guitart, 2012 p. 177). Para el autor el reto consiste en partir de estos fondos de identidad, que expresan los conocimientos previos, intereses y motivaciones del aprendiz, para que el docente pueda detectarlos, reconocerlos e incorporarlos como prácticas pedagógicas y recursos legítimos dentro de las instituciones educativas. En otras palabras, se espera aumentar la motivación, implicación y rendimiento escolar a partir del uso de los conocimientos, destrezas e intereses de los alumnos y alumnas que expresan fondos particulares de identidad. Lo que sin duda implica una continuidad entre los diferentes contextos de aprendizaje del alumnado.

El propósito de la incorporación de los "fondos de conocimiento e identidad" en el proyecto es facilitar que el docente sea palanca de cambio en el desarrollo de aprendizajes significativos en los estudiantes a partir de una vía didácticamente inclusiva. La historia oral es un excelente instrumento que enriquece la consideración de las experiencias de vida de las personas como fuentes válidas de conocimiento. El vínculo entre los fondos de conocimiento e identidad y la historia oral queda establecido en la medida en que se logre producir una continuidad entre el contexto educativo formal con aquellos aspectos socioculturales que el alumnado comparte con otros nichos de aprendizaje no formal.

El reto que se plantea, es relacionar las experiencias de vida de los estudiantes recogidas a través de las historias orales y convertirlas, bajo un cuidado proceso de reflexión y análisis, en actividades educativas formales considerando la diversidad cultural del alumno. En este sentido, la continuidad se establece al momento en que el profesor reconstruye la historia de vida de sus alumnos y logra aprender cuáles son sus aspectos más significativos. Lo que posibilita que las actividades de enseñanzaaprendizaje establezcan continuidades y, por ende, mejores niveles de progreso académico en el alumnado. Se espera, además, que estas prácticas educativas, a su vez, hagan del profesor en formación un aprendiz de sus propios alumnos a través de un cambio profundo en las relaciones de enseñanza-aprendizaje desarrollando una actitud más reflexiva en los docentes sobre sus propias prácticas. Perspectiva a la que hemos denominado "didáctica cultural inclusiva" (DCI). Visto así, tanto alumno como profesor podrán desarrollarse como actores críticos de su propia actividad educativa.

Por lo anterior, el objetivo de la primera parte del estudio es documentar, a partir de los grupos focales, qué ideas y creencias tienen los docentes en formación acerca de tres temáticas trascendentales sobre las familias de sus alumnos, el 
aprendizaje y las unidades didácticas que se desarrollan en el aula. Nos interesa hacer emerger las creencias previas sobre dichos temas educativos, justificar la implementación del programa "fondos de conocimiento e identidad" en el caso que existan discontinuidades educativas y poder, al finalizar la investigación, documentar el cambio de creencias por parte de los docentes una vez hayan participado en el desarrollo global del proyecto de innovación educativa.

\section{Método}

Para conducir la investigación se eligieron el enfoque cualitativo y el paradigma educativo sociocultural, cuyo propósito es el estudio de las personas a partir de sus prácticas sociales en su situación histórica y social (Pérez-Campos y Yoseff-Bernal, 2012). Además, el método de investigación basado en el diseño (DBR) utilizado en este estudio resultó compatible con el paradigma seleccionado teniendo como propósito el desarrollo de una investigación capaz de incrementar la comprensión acerca del fenómeno fortaleciendo el desarrollo de aplicaciones útiles para la formación inicial del profesorado, especialmente en las prácticas que se desarrollan en el contexto de la vida real.

La DBR se define como la metodología diseñada para incrementar el impacto, la transferencia y el tránsito de la investigación educativa en la práctica educacional (Anderson y Shattuck, 2012). La DBR, se constituye como un método sensible al contexto social de modo que toma en cuenta el fenómeno que se desarrolla en contextos naturales así como en la generación de ciertos datos empíricos (Barab y Squire, 2004; Anderson y Shattuck, 2012). Además, los resultados obtenidos tienen un mayor potencial para influir mejor en la práctica educativa, buscando que la investigación produzca cambios demostrables a nivel local. Por ello, se pone especial énfasis en que el investigador documente el aprendizaje generado dotando de mayor garantía al marco teorético planteado. En la DBR, el término validez se mide a partir de los cambios producidos (Barab y Squire, 2004). Estos cambios y consecuencias se consideran evidencias que apoyan la validez, de tal manera que los resultados puedan ser usados de modo efectivo en la evaluación, información y mejora de la práctica en, al menos, los contextos de intervención.

Una de las fortalezas de la DBR es la posibilidad de que los investigadores puedan ir ajustando varios aspectos del diseño de la investigación permitiendo al investigador analizar y generar teoría en contextos naturales. Siendo muy conscientes de las características locales del contexto investigado, la DBR va en la dirección de generar y avanzar hacia un conjunto de constructos teoréticos que transciendan el contexto particular en que han sido generados, seleccionados y redefinidos a partir del desarrollo de modelos que respondan a cómo los humanos piensan, saben, actúan y aprenden (Barab y Squire, 2004). Además, la colaboración entre investigadores y participantes en la DBR es un rasgo característico en la DBR. La selección y construcción de la intervención que se diseña a partir de las necesidades del contexto local, se apoya en la literatura, teorías, prácticas afines y se diseña con el fin de superar o mejorar una práctica. 
Por último, la DBR abre la posibilidad de incorporar múltiples metodologías y una variedad de técnicas y herramientas de investigación. El uso y selección por parte de los investigadores de métodos diferentes se lleva a cabo en función de "la necesidad, aplicando sus descubrimientos a la realidad que es a la vez plural y desconocida" (Maxcy 2003, p. 59).

Por ello, hacer uso del instrumento de grupos focales en un contexto educativo real (los salones de clase de la Escuela Normal de Texcoco) con el grupo de segundo año dentro de las actividades formativas de la licenciatura de Historia se considera coherente con la metodología planteada. Es importante para ello documentar las creencias iniciales de los docentes en formación para poder ajustar mejor el diseño de investigación planteado por los investigadores.

\section{Características y selección de participantes}

Participaron en el estudio un total de 16 maestros en formación del segundo curso de la Licenciatura en Educación Secundaria en Historia, de los cuales 11 eran mujeres y 5 hombres. El contexto de intervención fue la Escuela Normal de Texcoco, México. La selección de la Escuela Normal fue motivada por el hecho de que uno de los psicólogos-investigadores es a su vez profesor de normalistas en dicha institución y por la existencia del acuerdo de estancia de intercambio de experiencias académicas y de investigación educativa entre la Escuela Normal de Texcoco, Estado de México y la Universidad de Girona, España. Los participantes estaban matriculados en la asignatura de "Adolescencia II" y a la asignatura de "Observación y acercamiento a la práctica". Los 16 alumnos eran la totalidad de maestros en formación del curso académico 2014/2015. La participación en el proyecto se presentó como voluntaria y al margen de las evaluaciones académicas de las asignaturas en curso. Una vez aceptaron participar en el proyecto, se les hizo firmar a los 16 alumnos participantes un consentimiento escrito en que se comprometían a llevar a cabo la investigación en toda y cada una de las tres fases. El estudio fue dirigido por dos psicólogos-investigadores y con la participación necesaria de la maestra titular de la asignatura de "observación y acercamiento a la práctica".

\section{Instrumentos utilizados}

El instrumento metodológico de grupos focales ha sido el utilizado para documentar la concepción y significado de los profesores en formación sobre el contexto sociocultural y las necesidades de aprendizaje de los alumnos de secundaria. Por ser una herramienta eficaz en fases exploratorias en la construcción de la hipótesis de investigación para poder desarrollar un estudio de mayor envergadura, su selección se entiende como la más adecuada a la hora de identificar la existencia de discontinuidades entre los contextos educativos formales e informales y los contextos socioculturales del alumno.

Entendemos por grupos focales aquellas reuniones de individuos seleccionados por los investigadores para discutir y elaborar, desde la experiencia personal, una temática o hecho social que es objeto de investigación (Powell y Single, 1996). Una de las características del instrumento es el poder captar la profundidad sobre las 
diferentes cuestiones y ámbitos que emergen en el contexto de la entrevista grupal. Yendo en busca de las creencias y significados, nuestro interés en utilizar esta técnica es poder profundizar, seleccionando el grupo de docentes en formación, en sus realidades, experiencias, valores, costumbres, expectativas, actitudes, emociones y vivencias referentes a los mundos de vida de sus alumnos; arraigados de forma fundamental en los contextos culturales y sociales en donde se lleva a cabo la práctica educativa.

El proceso de le entrevista grupal de grupos focales se inicia a partir de un acuerdo entre los participantes en la entrevista y el investigador (o investigadores), en relación a los propósitos de la investigación. Se constituye como una modalidad de entrevista grupal abierta y estructurada tomando la forma de una conversación dirigida hacia el desarrollo de unas conclusiones grupales producto de la interacción y elaboración entre los participantes.

Aunque es el investigador quien guía la entrevista grupal y va incorporando las temáticas a medida que se va desarrollando la sesión, es necesaria una negociación con los participantes para asegurarnos que estos tengan una experiencia específica u opinión sobre las temáticas a tratar. Por ello, es necesario que el investigador elabore previamente un guión con aquellas preguntas o cuestiones que se quieren introducir a lo largo del proceso.

La elaboración del guión temáticas-preguntas parte de las experiencias personales y académicas de los entrevistados con el objetivo de hacer una exploración sistemática, pero no cerrada de la realidad. A continuación proponemos el guión de entrevista del tercer grupo focal sobre el ámbito temático de Secuencia Didáctica (SD):

\section{Guión Entrevista}

Temática: Secuencia Didáctica

- ¿Qué entienden ustedes por una planeación didáctica?

- La SD ¿cuál es el objetivo principal; para qué o quién se hace?

- ¿Con respecto a los contenidos y aprendizajes incluidos en la SD, estos contenidos quién los prescribe y los establece?

- ¿Las actividades como se seleccionan? ¿Estas actividades como se han seleccionado y diseñado?

- ¿Realmente la SD representa las necesidades de aprendizaje de los alumnos?

- ¿La planeación te está ayudando a desarrollar tus competencias de enseñanza y el desarrollando competencias de aprendizaje en los alumnos?

- ¿La SD está conectada o desconectada con las necesidades de aprendizaje del alumno?

- ¿Se hace ese reconocimiento de las necesidades del alumno, familia y comunidad en la planeación? ¿Se tienen las herramientas?

- ¿Hay algún instrumento que les ayude a conectarse directamente con el alumno?

- ¿Qué aprendizajes han generado en sus alumnos y en ustedes como futuros profesores?

Fuente: Elaboración propia.

\section{Figura 1.}

Las tres sesiones de entrevista grupal se desarrollaron por medio de la técnica de grupos focales. La intención ha sido que todos los docentes en formación participen 
de manera activa en la búsqueda de sentidos sobre aquello que se preguntaba. La dinámica de diálogo, en los grupos focales, fue empática y registrada en audio. La dirección de las entrevistas se llevó a cabo por un investigador-moderador mientras que otro investigador hacía el papel de "relator" tomando nota del comportamiento del grupo a partir de sus reacciones y comunicaciones no verbales. La identidad de los participantes ha sido resguardada bajo el principio del anonimato por lo que al momento de presentar algunas de las respuestas se ha de entender que corresponden a este grupo de 16 profesores en formación.

La primera sesión de investigación buscaba comprender qué, dónde y con quién aprendían los alumnos de secundaria. El propósito fue comprender cómo se definía el papel del alumno, el profesor y la escuela. La segunda sesión consistió en indagar sobre los contextos de vida y aprendizaje por los que transitaban los alumnos, tales como la familia y la comunidad y si éstos eran recursos válidos de aprendizaje. Finalmente, en la tercera sesión se les preguntó sobre la SD, específicamente sobre sus componentes así como de las actividades que la conformaban. Nuestro interés fue conocer hasta qué punto, las SD, estaban conectadas con las experiencias de vida e intereses de los alumnos.

El análisis de los datos se llevo a cabo a partir de las transcripciones de las grabaciones de cada uno de los grupos focales y, junto a las notas de campos y los comentarios reflexivos, conformaron los datos iniciales para su posterior análisis. Cada transcripción de grupo focal fue dividida en subcategorías en que se ordenaban las creencias y significados del grupo acerca de aquella categoría en cuestión. De este modo podíamos identificar cuáles eran los conceptos e ideas clave que imperaban en el grupo sobre las diversas cuestiones tratadas. Lo que a continuación se incorpora en el apartado de procedimiento son extractos recogidos de las intervenciones de los grupos focales que expresan las percepciones de los docentes en formación en torno a aquellas funciones que fueron analizadas como el significado sobre las discontinuidades entre la educación formal y los mundos de vida del alumno.

\section{Resultados}

En este trabajo se presentan los datos referentes a las tres sesiones de grupos focales del primer ciclo de la investigación. Bajo la lógica de la DBR es necesario tener datos que nos ayuden a redefinir los objetivos de todo el conjunto de la investigación así como rediseñar las fases de intervención. Los datos presentados a continuación son una selección de las transcripciones textuales de los grupos focales. Se hizo una depuración con el propósito de seleccionar las opiniones más significativas de los participantes, agrupándolas de la siguiente forma: a) relación entre profesoresalumnos; b) relación escuela-familia; c) definición de alumno y d) continuidadesdiscontinuidades didácticas.

Sobre la concepción del alumno tenemos:

“En las prácticas de observación pude observar mucho talento [...] no en cuanto al aprendizaje del currículum sino en cuanto a las habilidades que pueden utilizar en la escuela como [...] dibujar o hacer cosas creativas". 
"Los alumnos [...] están interactuando entre ellos, [...] se están contando cosas, sin darse cuenta están aprendiendo. Esos aprendizajes [...] son más significativos que muchos de los aprendizajes que les da el profesor".

"El alumno aprende todos los días y en todos los lugares; pero es importante la guía de alguien para que esos aprendizajes no se tornen negativos".

"Qué son capaces de hacer los alumnos...todo y nada. Todo, [...] sólo si tienen la necesidad de aprender algo. Si no tienen la necesidad no hacen nada. Es tan triste la situación [...] son como esponjas que esperan el conocimiento del maestro. Y eso es limitante $[\ldots]$ porque no crea autonomía".

"El alumno ha de cumplir con ciertas reglas y normas. El alumno ha de comportarse porque algo le están enseñando en la escuela. El alumno ha de responder a la sociedad porque por algo va a la escuela. Yo creo que en las escuelas los alumnos se sienten más prisioneros de la escuela que en otros lugares. Ellos han de cumplir con un reglamento (uniforme, corte de cabello, una manera de hacer). Ellos han de ser como la presentación de la escuela, y esa presentación en ellos significa no ser yo mismo".

"El alumno está determinado como sujeto dentro del sistema educativo [...] pero el alumno es persona e individuo y tiene su propia interpretación del contexto. El alumno aprende cuando está en interacción con ese contexto y con quien lo rodea. Lo más importante para mí en el [...] aprendizaje es el para qué; qué hago con ese aprendizaje".

"Ellos aprenden de todo, pero quizá no todo es funcional o aplicable en la escuela. Aprenden en la escuela, comunidad y sus amigos. [...] Con los diferentes eventos que hubo en el país de trata que el estudiante sea motor del cambio [...] El estudiante ha de ser creador y generador de nuevas cosas".

Los resultados en esta área sugieren que el alumno es un actor activo que interacciona con sus pares, familia y comunidad. Los docentes lo entienden como un sujeto que posee capacidades aprendidas en contextos de vida más cercanos. Es decir, el alumno aprende de todos y en todas partes pero es necesaria su orientación para que esos aprendizajes sean bien utilizados. Si bien existe la consciencia de que el aprendizaje es un hecho compartido por diferentes agentes y contextos educativos, no se especifica ni el grado, ni la forma en que la escuela puede incidir en ellos para desarrollar experiencias educativas significativas en los alumnos. En relación a los motivos de aprendizaje de los alumnos, los profesores en formación son ambiguos al entender a sus alumnos como personas que se adaptan "convenientemente". Pareciera como si los alumnos sólo se implican a aprender si les conviene; especialmente en la escuela. Los docentes ven este fenómeno como algo impersonal y no se ven como responsables en facilitar las condiciones adecuadas para generar motivación en sus alumnos. Finalmente, los docentes creen que el alumno no desarrolla todo su potencial en la escuela y se ve limitado en la toma de decisiones a consecuencia de su corta edad y experiencia. A raíz de lo analizado se puede llegar a entender al alumno como un ser activo pero en la medida en que sus circunstancias lo requieran. Entendemos que falta una profundización por parte de los docentes sobre lo que significa el "ser alumno" así como qué determina su participación en el aula como ser crítico y activo en el desarrollo de su propio aprendizaje. 
Sobre la relación profesor-alumno tenemos:

\begin{abstract}
"Qué sería un alumno si solo lo dejamos con lo que aprende de sus compañeros o lo que aprende de sus padres. Sería una educación mediocre. No tendríamos personas con un aprendizaje científico [...] ¿para qué estamos aquí (los docentes)? ¿Para qué son las escuelas? Yo creo que las escuelas tienen el objetivo de formar personas con un conocimiento científico".
\end{abstract}

"Conocer a tu alumno, a quién tienes en frente porque son personas, tienen gustos y tienen intereses y tú tienes que buscar la manera de crear la confianza (hablar sobre los videojuegos y decirle que puede relacionarlo con las matemáticas) es importante decírselo no de manera formal o técnica sino con un vocabulario informal para que ellos digan: pues sí que tiene razón. Entonces sí que es importante esto porque me está ayudando [...] Y también cómo fomentar que ellos puedan desarrollar aspiraciones (como ser el programador de algún videojuego) y para ello necesitan desarrollar ciertos conocimientos que se dan en la escuela".

"Estoy de acuerdo en que el alumno aprende en base a sus necesidades e intereses. El maestro selecciona la información [para] el alumno. Pero el alumno, de esa información, va a seleccionar lo que él quiere para sí mismo. La selección de información puede ser tanto académica como para su vida. Por ello todo depende del alumno y la información que requiere para su vida. La figura del maestro es más como una guía sobre la información que le da al adolescente".

"Yo entiendo que un alumno es una personita que aprende y enseña a la vez. [...] va a interactuar con el maestro, le va a enseñar algo nuevo al maestro. Yo vi en las prácticas cómo los alumnos enseñaron a la maestra a conectar una simple conexión de sonido [...] el alumno le enseñó cómo hacer cosas al maestro".

Los resultados en esta área sugieren que la relación profesor-alumno es fundamental para los docentes. En gran parte debido a que el profesor es quien puede seleccionar lo que es conveniente de ser aprendido. No obstante, el alumno es quien define con qué se queda de esa información. Por otro lado emerge la idea que los alumnos pueden enseñar ciertos aprendizajes al profesor. En las creencias de los profesores existe la postura tradicional de entender quién es más importante para el desarrollo de los aprendizajes en el alumno; si el alumno, el docente o los contenidos. Hace falta un mayor trabajo sobe la teoría para redefinir la relación profesor-alumno que establecen los docentes en formación de tal manera que se incluyan los proceso de mediación y negociación entre ambos. Además poner el énfasis en la posibilidad que los alumnos puedan enseñarle a su vez al profesor, modifica la perspectiva jerárquica y tradicional de los procesos de enseñanza y aprendizaje y legitima al alumno, familias y comunidades como uno de los más importantes recursos para el desarrollo educativo en el aula (Moll, 2014). Esto implica pensar en una posible unidad entre la enseñanza y el aprendizaje.

Sobre la relación escuela-familia tenemos:

"El problema es que se les cede mucho la responsabilidad a la escuela como el único lugar donde tienen que aprender, pero el lugar donde más conocimientos generan es en casa. Son los fundamentos donde ellos pueden insertarse a la educación. Porque si tu no le enseñas a un niño como debe de aprender, como ha de comportarse [...] 
El maestro ha de ser el mediador del conocimiento, porque si no lo es pues... ¿Qué estamos haciendo aquí? Si el alumno aprende por el solo pues ¿Qué hacemos aquí [...] estudiando cómo maestros?".

"Creo que en todo momento se aprende pero hay cosas que aprendemos y después las dejamos de practicar. Es un aprendizaje inútil y lo tiramos a la basura. Si se aprende algo en la escuela es importante que se retomen en las familias. [...] Y que también en la escuela es importante que se retomen los valores que debe tener una persona íntegra".

"Pero la escuela no es nada más la responsable, tiene parte de responsabilidad junto a la familia y la sociedad. [...] En las prácticas veo que los padres van a la escuela por que están castigados y muy pocos para saber cómo están sus hijos. Pero, de esos hijos que veo que sus padres están interesados es porque hay algo que los impulsa a los muchachos para que salgan; yo estoy viendo por mi familia y por mi porvenir. Se les está dejando la responsabilidad a otras personas y nadie quiere asumir la responsabilidad".

"Les preguntan los padres por las notas y no por los aprendizajes. Creo que ello no tiene mucho sentido. En cuento a los aprendizajes ellos (los alumnos) no son conscientes de que están aprendiendo algo. Solo viven esa experiencia y ya".

Sobre esta área tenemos que el vínculo de la familia con la escuela es deseado ya que puede proveer de una relación positiva. Sin embargo, no se clarifica el grado de relación e implicación de cada uno de los contextos educativos en el desarrollo del adolescente. Pese a ello, una de las figuras que tiene un mayor peso en la construcción de dicho vínculo es el profesor. Esto sugiere que la continuidad entre familia y escuela se supedita en gran medida a las acciones de los profesores. Aún así, existen dudas sobre cuáles son las funciones que pueden tener las familias en el aprendizaje del alumno dentro de la escuela. Parece que falta clarificar y reforzar continuidades entre la escuela y la familia (o comunidad) ya que no se trata únicamente de establecer comunicación solamente el día que se reparten las calificaciones sino que es necesaria una acción conjunta que incida en el fortalecimiento del progreso académico del alumno.

Sobre la relación continuidades-discontinuidades didácticas tenemos:

"Nosotros no desarrollamos lo que el alumno necesita sino lo que el plan nos está diciendo que desarrollemos. Eso es lo que nos complica mucho al ir a practicar"

"Para organizar la SD debemos conocer algunas características de los alumnos: donde vive, dedicación padres, los intereses que él tiene y de ahí la SD es la base para que el docente imparta la clase de acuerdo a aquello que quiere que los alumnos aprendan con lo que ya conoce del alumno"

"Yo creo que las herramientas que nosotros tendríamos que acoplar serían las propias planeaciones didácticas. Qué es lo que el maestro cree que está haciendo y qué es en realidad lo que el alumno hace"

"Conocer al alumno los primeros días y en base a ello planificar. Invertido en conocer"

"Esa herramienta a buscar y desarrollar creo que no es tal vez trabajo de uno solo. Creo que es un trabajo en equipo incluyendo toda la comunidad educativa (...) en equipo podemos diseñar esa herramienta para poder crear esa confianza con el alumno y una comunicación eficaz" 
Sobre este ámbito podemos identificar que los docentes en formación verbalizan que existe una distancia entre los planes y secuencias didácticas que desarrollan los profesores y las necesidades del alumno. Es más, esta distancia generada es vista no tan solo como una complicación hacia los alumnos a los que se imparte la lección, sino una dificultad que padecen los propios docentes a la hora de elaborar e implementar su práctica. Los docentes en formación, al aplicar una SD desvinculada de la realidad cotidiana, se sienten como docentes no competentes al no poder generar conocimientos significativos en sus alumnos.

Por lo anterior, podemos afirmar que los docentes en formación identifican las discontinuidades existentes entre la didáctica escolar y lo que el alumno conoce o le interesa. Además, son los mismos docentes quienes indican las posibles soluciones para mitigar esta distancia a través de un mayor conocimiento de los alumnos, familias, comunidades y contextos culturales. Es de este modo que la SD se plantea como una oportunidad para mejorar la relación didáctica entre alumno y profesor reforzándola con el conocimiento explícito de los alumnos para mejorar el desarrollo de los aprendizajes. Este trabajo de mejora e innovación educativa, además, no se puede llevar a cabo solamente por el docente sino que implica a toda la comunidad educativa; es un trabajo conjunto que compete a todos. De todas formas queda mucho por avanzar en definir cuál es el grado de implicación de cada agente educativo en la mejora de la propuesta educativa en el centro, ya que los docentes en formación no acaban de identificar soluciones pragmáticas y se quedan más en la superficialidad de sus afirmaciones.

\section{Conclusiones}

Los estudios cualitativos nos permiten poder acercarnos a comprender en profundidad los relatos de grupos particulares en sus contextos particulares siendo sensible a elementos culturales, lingüísticos, de clase o grupo social. Una de las contribuciones del presente artículo ha sido poder acercarnos a las perspectivas y puntos de vistas de los docentes en formación en la Escuela Normal de Texcoco para indagar sobre cuestiones tan fundamentales como qué entienden ellos por educación, qué es ser alumno, qué es el aprendizaje y dónde se desarrolla, qué papel tiene el alumno, la familia y la comunidad en la educación escolar o bien qué hay que mejorar para generar auténticos aprendizajes significativos en nuestros estudiantes. El análisis de los discursos docentes nos permite acercarnos a las necesidades e inquietudes del docente sobre su práctica para poder así desarrollar procesos de innovación educativa más alineados con la vida real de los docentes en formación.

Los resultados permiten ilustrar la desconexión del profesor en formación con los elementos culturales del contexto educativo que envuelven a sus alumnos. Situación que evidencia discontinuidades entre los contextos culturales de aprendizaje del alumnado y la escuela como institución. Ello se materializa en un desdibujamiento de la ecología del aprendizaje debido a la desvinculación de las actividades de planeación didáctica y las necesidades de aprendizaje de los alumnos. Esta conclusión alude al problema que hemos venido describiendo: la falta de relación entre los contextos educativos formales e informales y por lo tanto la inadecuada relación de conocimientos entre los diferentes contextos socioculturales del alumno. Esto, no 
sólo supone una falta de consistencia en el proceso de enseñanza aprendizaje, sino toda una discontinuidad entre las actividades que se realizan en el entorno educativo formal particularmente lo que corresponde al proceso de formación inicial.

Los docentes en formación, desde sus creencias y significados compartidos señalan la necesidad de construir vías de renovación, tanto en la relación directa con los alumnos de educación secundaria como en las mismas aulas de la Escuela Normal. Lo que implica la reflexión de los procesos de enseñanza-aprendizaje, en el contexto de las prácticas docentes, así como un análisis de las dinámicas y condiciones en las que se desarrolla su propia formación inicial.

Por lo anterior, entendemos que los fondos de conocimiento e identidad y la DCI pueden ser una vía pertinente de continuidad educativa y una metodología válida para superar las necesidades que los docentes en formación tienen en sus prácticas educativas cotidianas. La postura aquí defendida, entiende el aprendizaje como un proceso social que está ligado a diferentes contextos que afectan a la vida del estudiante. Son estos recursos y prácticas culturales que emanan de los contextos de vida de los alumnos aquellos elementos que median en el desarrollo del pensamiento (González et al., 2005). Las relaciones sociales, las circunstancias sociohistóricas, las experiencias de vida, las narraciones de las familias y comunidades son residuos intelectuales que mediados adecuadamente dentro de las actividades, didácticas y prácticas escolares por parte de los docentes pueden significar aprendizajes más alineados con los intereses y necesidades de sus alumnos. El hecho de reconocer al estudiante y a su entorno como portador de recursos válidos para la escuela plantea a su vez un cambio del rol y la responsabilidad en el salón de clase. El alumno es ahora un actor activo y responsable de su propio aprendizaje revertiendo el rol tradicional del maestro de transmisor de conocimientos. El docente se convierte en aprendiz de los mundos de vida de su alumnado reconociendo los recursos culturales de éste como fuentes legítimas de conocimiento. Desde este planteamiento una de las herramientas pedagógicas que puede enriquecer la interacción profesor-alumno a partir del reconocimiento de los fondos culturales son las SD. Una SD sensible a los fondos culturales de los alumnos puede generar una mayor continuidad en el proceso de enseñanza-aprendizaje en la medida en que se fundamente en el reconocimiento de conocimientos y aprendizajes validos más allá del contexto de educación formal. En el programa fondos de conocimiento e identidad ya se identifica la resignificación de las SD por parte de los docentes como un elemento clave para la transformación de la práctica educativa en el aula; incorporando los recursos culturales de los alumnos y generando un proceso de reflexión conjunto entre docentes e investigadores en lo que se han venido a llamar los grupos de estudio (González et al., 2005).

Para concluir nos gustaría señalar algunas posibles líneas de investigación emergentes en relación a la formación inicial del profesorado, la articulación y extensión de la práctica escolar a otras ecologías y contextos de socialización y el estudio de la reflexión del profesorado.

Por lo que compete a la formación inicial del profesorado, el presente artículo sobre las creencias de los docentes en formación y los subsiguientes ciclos se estructuran en un primer proyecto piloto que ha ido superando a medida que se iba planteando y desarrollando las diferentes problemáticas y necesidades que se encontraba 
de una manera incipiente. Pese a ello, la incorporación dentro de las prácticas educativas de contextos culturales tan sensibles como la familia y la comunidad puede convertirse en un instrumento válido para favorecer la cohesión social, aproximar la escuela al territorio y hacer emerger el sentido en la institución escolar. El desarrollo del proyecto con la implicación de la comunidad educativa de la Escuela Normal de Texcoco y el consentimiento y participación de las escuelas de secundaria de prácticas, así como de las familias y comunidades de los alumnos se constituye como uno de los retos a plantear para futuras intervenciones.

Por otra parte, se percibe la necesidad en renovar la formación docente puesto que el sistema de educación normalista, aún a pesar de la reforma 2012, es escenario de tensiones y resistencias que reflejan un modelo educativo tradicionalista. Motivo para pensar que la relevancia de este sistema educativo, así como su influencia en la sociedad mexicana, es de valor estratégico para el futuro del país y por lo tanto aún más necesaria su transformación. Aunque los datos obtenidos en esta investigación no son generalizables per se a los docentes en formación de otras Escuelas Normales del Estado de México, pensamos que el marco teórico y metodológico de los fondos de conocimiento e identidad y la DCl presentan propuestas lo suficientemente abiertas y flexibles para que su desarrollo, a partir de la intervención en la Escuela Normal de Texcoco, pueda ser adaptado a cada contexto a partir de las necesidades reales del profesorado. El desarrollo futuro de investigaciones que planteen la metodología de la $\mathrm{DCl}$ en procesos de formación inicial del profesorado acorde a la nueva reforma educativa de las Escuelas Normales se plantea nuevamente como una de las propuestas de futuro.

Finalmente, el contexto de intervención del programa de fondos de conocimiento e identidad se ha focalizado en la formación inicial con docentes en formación de escuelas normales. De todos modos, poder aproximarnos a los profesores de secundaria que ya están en el campo profesional a partir de la reflexión en la práctica docente se postula como una estrategia que pueda producir el cambio en el sentido y significación a partir de la innovación en las actividades de enseñanza-aprendizaje.

Aún falta recorrer un largo camino en la investigación educativa, desde la óptica de los fondos de conocimiento e identidad, pues esta perspectiva es aún incipiente en el contexto mexicano. Cualquier planteamiento al respecto será positivo en la medida en que acortemos la discontinuidad de nuestra comprensión sobre los contextos que conforman nuestra ecología del aprendizaje.

\section{REFERENCIAS BIBLIOGRÁFICAS}

Anderson, T. y Shattuck, J. (2012). Design-Based Research A Decade of Progress in Education Research? Educational researcher, 41, 16-25.

Barab, S. y Squire, K. (2014). Design-Based Research: Putting a Stake in the Ground. The Journal of the Learning Sciences, 13, 1-14.

Barron, B. (2010). Conceptualizing and Tracing Learning Pathways over Time and Setting. Yearbook of the National Society for the Study of Education, 109, 113-127.

Bronfenbrenner, U. (1987). La ecología del desarrollo humano. Experimentos en entornos naturales y diseñados. Barcelona: Paidós. 
Castells, M. (1994). Nuevas perspectivas críticas en Educación. Barcelona: Paidós.

Cole, M. y Engeström, Y. (2001). Enfoque histórico-cultural de la cognición distribuida. En Gavriel Salomon (coord.), Cogniciones distribuidas. Consideraciones psicológicas y educativas. Buenos Aires: Amorrortu, 23-75.

Coll, C. (2010). Enseñar y aprender en el mundo actual: desafíos y encrucijadas. Pensamiento Iberoamericano, 7, 44-66.

Coll, C. (2013). El currículo escolar en el marco de la nueva ecología del aprendizaje. Aula de Innovación Educativa, 219, 31-36.

Coll, C., Onrubia, J. y Mauri, T. (2008). Ayudar a aprender en contextos educativos: el ejercicio de la influencia educativa y el análisis de la enseñanza. Revista de Educación, 346, 33-70.

Diario Oficial de la Federación (2013). Ley General de Educación en Diario Oficial de la Federación. Recuperado de http://www.sep.gob.mx/work/models/sep1/ Resource/3f9a47cc-efd9-4724-83e40bb4884af388/ ley_general_educacion.htm.

Dirección General de Compilación y consulta del Orden Jurídico Nacional (2011). Ley General de Educación. Recuperado de http://www.ordenjuridico.gob.mx/leyes.php.

Dirección General de Educación Superior para Profesionales de la Educación (2014). Estadísticas ciclo escolar 2013-2014. Recuperado de http://www.siben.sep.gob. $\mathrm{mx} /$ pages/estadisticas_recientes.

Engeström, Y. (2014). Learning by expanding. An activity-theoretical approach to developmental research. Cambridge: Cambridge University Press.

Esteban-Guitart, M. (2012). Towards a multimethodological approach to identification of funds of identity, small stories and master narratives. Narrative Inquiry, 22, 173-180.

Esteban-Guitart, M. (2014). Funds of Identity. En Thomas Teo (Ed.), The Encyclopedia of Critical Psychology. New York: Springer.

González, N., Moll, L. y Amanti, K. (2005). Funds of knowledge: Theorizing practices in households, communities, and classrooms. Mahwah, NJ: LawrenceErlbaumAssociates.

Kozulin, A. (2000). Instrumentos psicológicos: la educación desde una perspectiva sociocultural. Barcelona: Paidós.

Lave, J. y Chaiklin, S. (2001). Estudiar las prácticas. Perspectivas sobre actividad y contexto. Buenos Aires: Amorrortu.

Lave, J. y Wenger, E. (2003). Aprendizaje situado: participación periférica legítima. México: UNAM, FES Iztacala.

Leontiev, A. (1984). Actividad, conciencia y personalidad. México: Cartago.

Maxcy, S. (2003). Pragmatic threads in mixed methods research in the social sciences: The search for multiple modes of inquirí and the end of the philosophy of formalism. En Abbas Tashakkori y Charles Teddlie (eds.), Handbook of mixed methods in social and behavioral research (pp. 51-89). Thousand Oaks, CA: Sage.

Moll, L. (2014). L. S. Vygotsky and Education. London: Routledge.

OCDE (2010). Competencias del siglo XXI para los aprendices del milenio en los países de la OCDE. Paris: Publicaciones OCDE.

OECD (2012). Connected Minds: Technology and Todays's Learners, Educational Research and Innovation. Paris: OECD Publishing. 
Pérez-Campos, G. y Yoseff-Bernal, J. (2012). Desarrollo psicológico. Un enfoque sociocultural. México: UNAM, FES Iztacala.

Powell, R. y Single, H. (1996). Focus groups. International Journal of Quality in Health Care, 5, 499-504.

Salomon, G. (2001). Introducción del compilador. En Gavriel Salomon (Comp.) Cogniciones distribuidas. Consideraciones psicológicas y educativas (pp. 11-22). Buenos Aires: Amorrortu.

Tedesco, J. C. (2011). Los desafíos de la educación básica en el siglo XXI. Revista Iberoamericana de Educación, 55, 31-47.

UNESCO (2005). Hacia las sociedades del conocimiento. Informe mundial de la UNESCO. París: Ediciones UNESCO. 